\title{
spektrum der augenheilkunde
}

\section{Editorial}

\author{
Helga Azem
}

C Springer-Verlag Wien 2012

Sehr geehrte Damen und Herren!

Sehr geehrte Kolleginnen und Kollegen!

Wir haben ein ereignisreiches und erfolgreiches Arbeitsjahr hinter uns, das wir mit der Jahrestagung der Österreichischen Ophthalmologischen Gesellschaft vergangenen Mai abgeschlossen haben. Dieses Jahr war von einem Umbruch geprägt - ein Umbruch im gesundheitspolitischen Umfeld Österreichs, aber auch ein Umbruch direkt vor der Türe des ophthalmologischen Standes.

Diesen sich abzeichnenden Umbruch haben wir als ÖOG zum Anlass genommen, einen Nachdenkprozess einzuleiten und begonnen, eine klare Strategie der Absicherung des Stellenwertes der Ophthalmologie in Österreich zu definieren und zu verfolgen - bereits mit ersten Erfolgen! Im Mittelpunkt unserer Überlegungen sind auf der einen Seite die Interessen der Augenärztinnen und Augenärzte, auf der anderen Seite die bestmögliche medizinische Versorgung der Patientinnen und Patienten gestanden. Die Entwicklung hin zu einem Paradigmenwechsel der Gesundheitsberufe, die gekürzten Budgets im angestellten und niedergelassenen Bereich und auch die immer enger bemessenen Rückerstattungen der Sozialversicherungen haben uns dazu gezwungen, im Interesse des augenärztlichen Standes und der Versorgung der Patienten zu reagieren und erste Akzente zu setzen.

Die Balance zwischen den effizienten Positionierungsbemühungen bei den Entscheidern im österreichischen System und breiter öffentlichkeitswirksamer Aufmerk-

OMR Dr. H. Azem $(\square)$

Engerthstraße 193/3, 1020 Wien, Österreich

E-Mail: helga.azem@augenzentrum-azem.at samkeit ist nicht leicht zu halten. Es muss ein Ausgleich zwischen den verschiedenen Zielgruppen gefunden werden, was mit den zur Verfügung stehenden finanziellen Mitteln eine herausfordernde Aufgabe ist. Diesen Spagat konnten wir retrospektiv mit Hilfe professioneller Hilfe ob der kurzen Aktionszeit gut meistern.

Eines muss uns allen klar sein: Es ist kein erstrebenswertes Ziel, die Berufsgruppe der Optiker und Optometristen $\mathrm{zu}$ verdrängen oder deren Berechtigung als wichtiger Bestandteil einer generalistischen Versorgung des Auges abzusprechen. Vielmehr wollen wir das Wohl der Patienten in den Mittelpunkt unserer Überlegungen stellen. Unserer Meinung nach ist dieses Wohl am besten gewährleistet, wenn das Auge vom Augenarzt untersucht wird. Nicht umsonst genießt jeder Ophthalmologe eine gesamtheitliche humanmedizinische Ausbildung. Wer, wenn nicht der Augenarzt, hat somit die Aufgabe und die Fähigkeit, aber auch die Verantwortung, das Auge medizinisch zu untersuchen.

Die Stärken des Optikers und des Optometristen liegen in der Messung und idealen Anpassung von optischen Sehhilfen. Optiker haben aber nicht die Ausbildung, das Auge medizinisch zu untersuchen und vorsorglich Krankheitsbilder zu diagnostizieren oder auszuschließen. Hier bestehen eindeutige Grenzen. Es gibt keine akzeptablen Gründe, diese Grenzen zu verwischen. Eine Zusammenarbeit beider Berufsgruppen ist mit Sicherheit die wertbringenste Form, um die medizinische Versorgung der Patientinnen und Patienten zu verbessern.

Liebe Kolleginnen und Kollegen, wir freuen uns, dass wir im letzten Jahr schon sehr viel Positives bewirken konnten. In zahlreichen Gesprächen mit Vertretern des Gesundheitssystem, der Politik, den Krankenkassen und weiteren konnten wir bereits eine Bewusstseinsände- 
rung bei den Entscheidern, bei den Ärzten, aber auch in der Bevölkerung bewirken. Das zeigt uns die Marktforschung, die wir im Rahmen der Jahrestagung präsentiert haben. Die zahlreichen Aktivitäten (Europäischen Forum Alpbach, Podiumsdiskussion, Pressekonferenz etc.) zeigen somit schon erste positive Wirkungen und weisen eine vielversprechende Tendenz für die Zukunft auf. Es ist nun unsere Aufgabe, diesen positiven Weg fortzusetzen und weitere Verbesserungen zu etablieren.

Wir müssen uns bewusst sein, dass die nun von uns getroffenen Entscheidungen gravierende Auswirkungen für die Zukunft unseres Berufstandes haben werden. Falls wir uns entscheiden, keine weiteren Schritte zu setzen, fällt die Zukunft unseres Berufstandes - und meiner Meinung nach auch die des Wohls der Patienten - in eine Hand, wo Entscheidungen ausschließlich aufgrund bloß vordergründiger Wirtschaftlichkeit getroffen werden.
Bei der Jahrestagung haben wir beschlossen, die Kampagne nur dann fortzusetzen, wenn die Mehrheit der österreichischen Ophthalmologen das befürwortet. Aus diesem Grund wird es im Herbst eine Urabstimmung geben, wo alle ÖOG-Mitglieder demokratisch mitbestimmen dürfen. Wir sind davon überzeugt, dass die Fortsetzung dieses schon erfolgreichen Weges der einzig richtige sein kann!

Ich freue mich auch darauf, weiter im Sinne der Ophthalmologie in Österreich mit Ihnen zusammenzuarbeiten und hoffe auf Ihre fortgesetzte Unterstützung!

Mit allerbesten kollegialen Grüßen,

Dr. Helga Azem

Präsidentin der öoG 